\title{
Disturbance Observer-Based Continuous Finite-Time Sliding Mode Control against Matched and Mismatched Disturbances
}

\author{
Ngo Phong Nguyen, ${ }^{1}$ Hyondong Oh $\mathbb{D},{ }^{1}$ Yoonsoo Kim $\mathbb{D}^{2},{ }^{2}$ and Jun Moon $\mathbb{D}^{3}$ \\ ${ }^{1}$ School of Mechanical, Aerospace and Nuclear Engineering, Ulsan National Institute of Science and Technology (UNIST), \\ Ulsan, Republic of Korea \\ ${ }^{2}$ Department of Aerospace and Software Engineering, Gyeongsang National University, Jinju 52828, Republic of Korea \\ ${ }^{3}$ School of Electrical and Computer Engineering, University of Seoul, Seoul, Republic of Korea \\ Correspondence should be addressed to Hyondong Oh; h.oh@unist.ac.kr and Jun Moon; junmoon.illinois@gmail.com
}

Received 12 March 2020; Accepted 24 April 2020; Published 28 May 2020

Academic Editor: Xianggui Guo

Copyright ( $) 2020$ Ngo Phong Nguyen et al. This is an open access article distributed under the Creative Commons Attribution License, which permits unrestricted use, distribution, and reproduction in any medium, provided the original work is properly cited.

\begin{abstract}
In this paper, we propose the disturbance observer-based continuous finite-time sliding mode controller (DOBCSMC) for inputaffine nonlinear systems in which additive matched and mismatched disturbances exist. The objective is to show the robustness and disturbance attenuation performance of the closed-loop system with the proposed DOBCSMC subjected to general classes of matched and mismatched disturbances. The proposed DOBCSMC consists of three main features: (i) the nonlinear finite-time disturbance observer to obtain a fast and accurate estimation of matched and mismatched disturbances, (ii) the nonlinear sliding surface to ensure high precision in the steady-state phase of the controlled output, and (iii) the continuous supertwisting algorithm to guarantee finite-time convergence of the controlled output and reduce the chattering under the effect of matched and mismatched disturbances. It should be noted that the existing approaches cannot handle time-varying mismatched disturbances and/or cannot guarantee faster finite-time stability of the controlled output. We prove that the closed-loop system with the DOBCSMC guarantees both finite-time reachability to the sliding surface and finite-time stability of the controlled output to the origin. Various simulations are performed to demonstrate the effectiveness of the proposed DOBCSMC. In particular, the simulation results show that the DOBCSMC guarantees faster convergence of the closed-loop system to the origin, higher precision of the controlled output, and better robustness performance against various classes of (time-varying) matched and mismatched disturbances, compared with the existing approaches.
\end{abstract}

\section{Introduction}

Sliding mode control (SMC) is a well-known robust control method owing to its attractive features such as quick transient response, fine robustness against disturbances and parameter uncertainties, and ease of implementation [1-5]. Since its introduction, the SMC has been extensively studied in the literature. The readers are referred to [1-5] and the references therein. However, we should note that most of the existing traditional SMC approaches in [1-5] are insensitive only to matched disturbances and cannot attenuate mismatched disturbances effectively $[6,7]$.

Mismatched disturbances exist in various control applications, and they may result in performance degradation of the control system [6-8]. In recent years, there has been a significant interest in studying SMC approaches with systems affected by both matched and mismatched disturbances (see [6-17] and the references therein). In general, the aforementioned SMC approaches for systems in the presence of matched and mismatched disturbances can be divided into the following three categories: (i) the integral sliding mode control (ISMC); (ii) the robust sliding mode control; and (iii) the disturbance observer-based sliding mode control.

The key idea of the ISMC approach is that the integral action is added to the sliding variable to force the state to the desired equilibrium point in the presence of mismatched disturbances. Several approaches have been developed based 
on the ISMC method for systems with mismatched disturbances in [9-12]. However, the ISMC handles the mismatched disturbances in a robust way, which implies that the nominal performance is sacrificed to achieve better robustness.

The second category mainly focuses on the combination of the SMC with other robust control methods such as the adaptive control approach, backstepping control method, and optimal control technique [13-17]. When integrating the SMC with the robust control theory to handle mismatched disturbances, the disturbance attenuation is achieved in a trade-off that sacrifices its nominal control performance.

Finally, for (iii), as noted in [6-8], a class of nonlinear disturbance observers has been integrated with the SMC, which possibly attenuates the effect of mismatched disturbances. In [6], the enhanced sliding mode control (ESMC) via the disturbance observer was proposed for nonlinear systems with mismatched disturbances. It should be noted that in [6], only the time-invariant mismatched disturbance can be handled. Moreover, in the sliding phase of the SMC, only the asymptotic stability is guaranteed instead of the finite-time stability. Note also that the chattering phenomenon still exists as the corresponding SMC is discontinuous. In [7], the authors developed a continuous dynamic sliding mode control (CDSMC) for systems with timevarying disturbances. Besides, the continuous nonsingular terminal sliding mode control (CNTSMC) for systems with mismatched disturbances was proposed in [8]. The higherorder sliding mode observer (HOSMO) scheme was used in the CDSMC and CNTSMC to provide a precise estimation of (time-varying) mismatched disturbances. Although the CDSMC and CNTSMC are continuous, the power reaching scheme included in their controllers may degrade the robustness of the closed-loop system $[18,19]$. Additionally, in $[7,8]$, the system behaves as a desirable reduced-order dynamics during the sliding-mode motion, not full-order dynamics, which may not ensure high-precision control $[20,21]$. We also note that the integrated HOSMO in $[7,8]$ produces a slow convergence rate when the initial estimation errors are relatively large $[22,23]$.

Note that the aforementioned SMC approaches cannot handle a general class of time-varying mismatched disturbances and/or cannot guarantee faster finite-time stability of the controlled output. In this paper, we address these two issues. Hence, unlike the existing approaches, our proposed method can be applied to a system that has both timevarying matched and mismatched disturbances, which guarantees faster finite-time stability of the controlled output. A detailed statement of the proposed approach is given below.

In this paper, we propose the disturbance observer-based continuous finite-time sliding mode controller (DOBCSMC) for input-affine nonlinear systems in which additive matched and mismatched disturbances exist. The main objective is to show the robustness and disturbance attenuation performance of the closed-loop system with the proposed DOBCSMC subjected to general classes of matched and mismatched disturbances. The proposed DOBCSMC consists of three main features:

(i) The nonlinear finite-time disturbance observer to obtain a fast and accurate estimation of matched and mismatched disturbances.

(ii) The nonlinear sliding surface to ensure high precision in the steady-state phase of the controlled output.

(iii) The continuous supertwisting algorithm to guarantee finite-time convergence of the controlled output and reduce the chattering under the effect of matched and mismatched disturbances.

As mentioned above, the existing approaches cannot handle time-varying mismatched disturbances and/or cannot guarantee faster finite-time stability of the controlled output. We prove that the closed-loop system with the DOBCSMC guarantees both finite-time reachability to the sliding surface and finite-time stability of the controlled output to the origin. Various simulations are performed to demonstrate the effectiveness of the proposed DOBCSMC. In particular, the simulation results show that the DOBCSMC guarantees faster convergence of the closedloop system to the origin, higher precision of the controlled output, and better robustness performance against various classes of (time-varying) matched and mismatched disturbances, compared with the existing approaches.

We should mention that our paper can be viewed as an extension of the results in [4-10]. Specifically, as stated in Table 1, the detailed comparisons are given as follows:

(1) The proposed DOBCSMC is an extension of finitetime SMC (FSMC) $[4,5]$ to the case of systems with mismatched disturbances.

(2) The DOBCSMC generalizes the results of $[6,9,10]$ to the case of time-varying mismatched disturbances.

(3) The DOBCSMC extends the results in $[6,9,10]$ to the finite-time stability of the controlled output with the continuous supertwisting control law.

(4) The DOBCSMC can guarantee finite-time stability of the controlled output, whereas the CDSMC [7] shows only asymptotic stability.

Besides, our paper is different from $[7,8]$ in that the DOBCSMC is proposed based on the supertwisting reaching scheme rather than the power reaching approach as in $[7,8]$; hence, with our DOBCSMC, it is possible to improve the robustness of the closed-loop system $[18,19]$. In addition, the DOBCSMC maintains the system in desirable slidingmode motion dynamics by means of the nonlinear sliding surface, which provides better control performance, compared with the sliding-mode motion in $[7,8]$ (see a related discussion in $[20,21])$. Finally, the nonlinear finite-time observer used in our DOBCSMC provides faster convergence rate, compared with the HOSMO in $[7,8]$ (see $[22,23]$ and the references therein).

The paper is organized as follows. Section 2 presents the problem formulation. Some useful lemmas are provided in 
TABle 1: Features of FSMC, ISMC, ESMC, CDSMC, and DOBCSMC

\begin{tabular}{lccc}
\hline & Controller & $\begin{array}{c}\text { Mismatched } \\
\text { disturbances }\end{array}$ & Stability \\
\hline FSMC [4, 5] & Continuous & N/A & Finite-time \\
ISMC [9, 10] & Discontinuous & Time-invariant & Asymptotic \\
ESMC [6] & Discontinuous & Time-invariant & Asymptotic \\
CDSMC [7] & Continuous & Time-varying & Asymptotic \\
DOBCSMC & Continuous & Time-varying & Finite-time \\
\hline
\end{tabular}

Section 3. In Section 4, we propose the DOBCSMC and show the finite-time reachability and stability. The simulation results are provided in Section 5. The concluding remarks are given in Section 6 .

\section{Problem Formulation}

Consider the following second-order input-affine nonlinear system subjected to matched and mismatched disturbances:

$$
\begin{aligned}
& \dot{x}_{1}=x_{2}+d_{1}(t), \\
& \dot{x}_{2}=f\left(x_{1}, x_{2}\right)+u+d_{2}(t), \\
& y=x_{1},
\end{aligned}
$$

where $x_{1}, x_{2} \in \mathbb{R}$ are states, $u$ is the control input, $y$ is the controlled output, $f\left(x_{1}, x_{2}\right)$ is a smooth nonlinear function, and $d_{1}$ and $d_{2}$ denote the mismatched and matched disturbances, respectively. Below, we sometimes write $d_{1}(t)$ and $d_{2}(t)$ to emphasize that the disturbances are timevarying.

The main goal of this paper is to design the disturbance observer-based continuous finite-time sliding mode controller for system (1) such that the proposed controller is able to drive the sliding variable to zero in finite time and the controlled output $y=x_{1}$ to zero in finite time despite the presence of time-varying matched and mismatched disturbances.

We now briefly introduce the existing traditional SMC, ISMC, and ESMC for a system affected by matched and mismatched disturbances.

2.1. Traditional SMC. For system (1), the sliding variable can be defined as

$$
s=x_{2}+c x_{1}
$$

where $c>0$ is the design parameter. Taking the derivative of (2) along (1) yields

$$
\dot{s}=f\left(x_{1}, x_{2}\right)+u+d_{2}+c\left(x_{2}+d_{1}\right) .
$$

By selecting $u$ as

$$
u=-f\left(x_{1}, x_{2}\right)-c x_{2}-K \operatorname{sign}(s),
$$

the dynamics in (3) can be rewritten as follows:

$$
\dot{s}=-K \operatorname{sign}(s)+c d_{1}+d_{2},
$$

where $K>0$ is the design parameter.
Hence, we can easily observe that if $K>c d_{1}^{*}+d_{2}^{*}$, where $\left|d_{1}\right| \leq d_{1}^{*}$ and $\left|d_{2}\right| \leq d_{2}^{*}$, the system state (1), which initially stays outside the sliding surface, will reach the sliding surface $s=0$ in finite time. Taking the condition $s=0$ in (2), the sliding motion can be expressed as follows:

$$
\dot{x}_{1}=-c x_{1}+d_{1}
$$

which implies that the controlled output $x_{1}$ cannot be driven to the origin, although the sliding variable $s$ is forced to zero in finite time [6]. This also happens in the finite-time SMCs studied in $[4,5]$. Therefore, it can be concluded that the traditional SMC cannot be directly applied to a control system subjected to mismatched disturbances.

2.2. Integral SMC. As mentioned in Section 1, the ISMC can be considered as an effective method to handle a mismatched disturbance. For system (1), the integral sliding variable can be defined as

$$
s=x_{2}+c_{1} x_{1}+c_{2} \int_{0}^{t} x_{1} \mathrm{~d} \tau
$$

where $c_{1}>0$ and $c_{2}>0$ are the design parameters. Taking the derivative of (7) along (1) yields

$$
\dot{s}=f\left(x_{1}, x_{2}\right)+u+d_{2}+c_{1}\left(x_{2}+d_{1}\right)+c_{2} x_{1} .
$$

By selecting $u$ as

$$
u=-f\left(x_{1}, x_{2}\right)-c_{1} x_{2}-c_{2} x_{1}-K \operatorname{sign}(s),
$$

the dynamics in (8) can be rewritten as follows:

$$
\dot{s}=-K \operatorname{sign}(s)+c_{1} d_{1}+d_{2},
$$

where $K>0$ is the design parameter. This shows that if we select $K>c d_{1}^{*}+d_{2}^{*}$, the system state (1), which initially stays outside the sliding surface, will reach the sliding surface $s=0$ in finite time. When the system in (1) operates in the sliding mode phase, we can obtain $[9,10]$

$$
\ddot{x}_{1}+c_{1} \dot{x}_{1}+c_{2} x_{1}=\dot{d}_{1},
$$

which implies that the controlled output $y=x_{1}$ can converge to zero if the mismatched disturbance has a constant steady-state value, i.e., $\dot{d}_{1}=0$.

The ISMC has been shown to be an attractive method to eliminate the effect of mismatched disturbances in (1). However, the ISMC can only work with a system affected by time-invariant mismatched disturbances [6]. In addition, the control input of the ISMC is discontinuous, which induces chattering in the system response.

2.3. Enhanced SMC. As introduced in Section 1, the ESMC in [6] is an alternative approach to attenuate mismatched disturbances. It shall be pointed out that the authors in [6] assumed that system (1) is only affected by a mismatched disturbance, i.e., $d_{2}=0$. Under this assumption, the sliding variable can be defined as [6]

$$
s=x_{2}+c x_{1}+\widehat{d}_{1},
$$


where $c>0$ is the design parameter and $\widehat{d}_{1}$ denotes the estimation of the mismatched disturbance $d_{1}$ by a disturbance observer. Then, the ESMC can be designed as follows:

$$
u=-f\left(x_{1}, x_{2}\right)-c\left(x_{2}+\widehat{d}_{1}\right)-K \operatorname{sign}(s),
$$

where $K>0$ is the switching gain to be designed.

By following the same procedure as in Theorem 1 of [6], we can show that system (1) with $d_{2}=0$ under control law (13) is asymptotically stable by using the proper controller and observer gains.

It should be noted that the ESMC could attenuate the mismatched disturbance without introducing adverse control effects such as a large overshoot and unsatisfactory settling time, as occurs with the ISMC [6]. However, the control law of the ESMC is still discontinuous, indicating that the chattering problem is unavoidable. Besides, with the ISMC and ESMC methods, only time-invariant mismatched disturbances can be considered and in the sliding phase of the SMC, only the asymptotic stability is guaranteed rather than the finite-time stability.

\section{Remark 1}

(i) In summary, to attenuate the effect of mismatched disturbances on the system's controlled output, the aforementioned control approaches make some conservative assumptions on the mismatched disturbance, where the mismatched disturbance is required to be a vanishing one in the traditional SMC approach and have a constant steady-state value in the ISMC and ESMC methods $[1-6,9,10]$. However, the disturbance in practical control applications may not satisfy these assumptions (see $[7,8]$ and the references therein). In this case, the traditional SMC, ISMC, and ESMC in $[1-6,9,10]$ cannot attenuate the effect of mismatched disturbances effectively.

(ii) All aforementioned problems motivate us to study the problem in this paper, where we propose the disturbance observer-based continuous finite-time sliding mode controller (DOBCSMC). In particular, the proposed DOBCSMC guarantees (1) finite-time estimation of the actual values of the matched and mismatched disturbances and their derivatives; (2) reachability to the sliding surface in finite time; (3) finite-time stability to the origin of the controlled output; and (4) robustness and high-accuracy control performance, despite the presence of timevarying matched and mismatched disturbances.

\section{Preliminaries}

In this section, we provide preliminaries to obtain the main results in Section 4. The control scheme to address the problem posed in Section 2 will be built from the following three parts: (i) the finite-time differentiator proposed in [22]; (ii) the finite-time sliding variable developed in [5]; and (iii) the supertwisting algorithm from [4].

The following lemma summarizes the results of the finite-time stability for the corresponding dynamical system of the study of Levant and Livne in [22].

Lemma 1 [22, Theorem 3.1]. Consider the following system:

$$
\begin{aligned}
& \dot{\sigma}_{0}=-\lambda_{0}\left|\sigma_{0}\right|^{n /(n+1)} \operatorname{sign}\left(\sigma_{0}\right)-\varrho_{0} \sigma_{0}+\sigma_{1} \\
& \dot{\sigma}_{1}=-\lambda_{1}\left|\sigma_{1}-\dot{\sigma}_{0}\right|^{(n-1) / n} \operatorname{sign}\left(\sigma_{1}-\dot{\sigma}_{0}\right)-\varrho_{1}\left(\sigma_{1}-\dot{\sigma}_{0}\right)+\sigma_{2}, \\
& \vdots \\
& \dot{\sigma}_{n-1}=-\lambda_{n-1}\left|\sigma_{n-1}-\dot{\sigma}_{n-2}\right|^{1 / 2} \operatorname{sign}\left(\sigma_{n-1}-\dot{\sigma}_{n-2}\right)-\varrho_{n-1}\left(\sigma_{n-1}-\dot{\sigma}_{n-2}\right)+\sigma_{n}, \\
& \dot{\sigma}_{n}=-\lambda_{n} \operatorname{sign}\left(\sigma_{n}-\dot{\sigma}_{n-1}\right)-\varrho_{n}\left(\sigma_{n}-\dot{\sigma}_{n-1}\right)-\frac{1}{L_{0}} f(t),
\end{aligned}
$$

where $\sigma_{0}, \ldots, \sigma_{n}$ are the scalar state variables, $\lambda_{0}, \ldots, \lambda_{n}$ and $\varrho_{0}, \ldots, \varrho_{n}$ are appropriate positive constants, $L_{0}$ is a proper positive constant, and the perturbation term $f(t)$ satisfies $|f(t)| \leq L_{0}$. Then, the system converges to the origin in finite time, i.e., it is finite-time stable.

The following lemma summarizes the results about the supertwisting algorithm in [4].

Lemma 2 [4, Theorem 2]. Consider the following system:

$$
\begin{aligned}
& \dot{y}_{1}=-k_{1}\left|y_{1}\right|^{1 / 2} \operatorname{sign}\left(y_{1}\right)+y_{2}, \\
& \dot{y}_{2}=-k_{2} \operatorname{sign}\left(y_{1}\right),
\end{aligned}
$$

where $y_{1}$ and $y_{2}$ are the scalar state variables and $k_{1}>0$ and $k_{2}>0$ are the design parameters. Then, the system is strongly globally asymptotically stable when $k_{1}, k_{2}>0$. In fact, the system converges to the origin in finite-time $T$ with $T \leq 2 V^{1 / 2}\left(y_{0}\right) / \gamma$, where $y_{0}$ is the initial state, $V\left(y_{0}\right)=\zeta^{T} P \zeta$ with 


$$
\begin{gathered}
\zeta^{T}=\left[\left|y_{1}(0)\right|^{1 / 2} \operatorname{sign}\left(y_{1}(0)\right), y_{2}(0)\right], \\
P=0.5\left[\begin{array}{cc}
4 k_{2}+k_{1}^{2} & -k_{1} \\
-k_{1} & 2
\end{array}\right], \\
\gamma=\frac{\lambda_{\min }^{1 / 2}\{P\} \lambda_{\min }\{Q\}}{\lambda_{\max }\{P\}}, \\
Q=0.5 k_{1}\left[\begin{array}{rr}
2 k_{2}+k_{1}^{2} & -k_{1} \\
-k_{1} & 1
\end{array}\right] .
\end{gathered}
$$

The following lemma states the results of the finite-time convergence of the corresponding differential equation in [5].

Lemma 3 [5, Proposition 8.1]. The following nth-order system is considered:

$$
\begin{aligned}
\dot{\sigma}_{l} & =\sigma_{l+1}, \quad l=1,2, \ldots, n-1, \\
\dot{\sigma}_{n} & =u,
\end{aligned}
$$

where $\sigma_{l}(l=1,2, \ldots, n)$ represent the states and $u$ is the control input. There is $0<\epsilon_{b}<1$ such that the origin is a globally finite-time stable equilibrium for every $1-\epsilon_{b}<\bar{\alpha}<1$ by the controller

$$
u=-\gamma_{1}\left|\sigma_{1}\right|^{\alpha_{1}} \operatorname{sign}\left(\sigma_{1}\right)-\cdots-\gamma_{n}\left|\sigma_{n}\right|^{\alpha_{n}} \operatorname{sign}\left(\sigma_{n}\right),
$$

where the scalars $\gamma_{1}, \ldots, \gamma_{n}$ are positive design constants such that the polynomial

$$
p^{n}+\gamma_{n} p^{n-1}+\cdots+\gamma_{2} p+\gamma_{1}
$$

is Hurwitz and the scalars $\alpha_{1}, \ldots, \alpha_{n}$ are chosen recursively as

$$
\alpha_{l-1}=\frac{\alpha_{l} \alpha_{l+1}}{2 \alpha_{l+1}-\alpha_{l}}, \quad l=2, \ldots, n
$$

with $\alpha_{n+1}=1$ and $\alpha_{n}=\bar{\alpha}$.

Lemma 4 [24, Lemma 2]. For $x \in \mathbf{R}$ and $y \in \mathbf{R}$, if $\varepsilon_{1}>0$ and $\varepsilon_{2}>0$, then

$$
|x|^{\varepsilon_{1}}|y|^{\varepsilon_{2}} \leq \frac{\varepsilon_{1}|x|^{\varepsilon_{1}+\varepsilon_{2}}}{\varepsilon_{1}+\varepsilon_{2}}+\frac{\varepsilon_{2}|y|^{\varepsilon_{1}+\varepsilon_{2}}}{\varepsilon_{1}+\varepsilon_{2}} .
$$

\section{Main Results}

In this section, we present the main results of the paper. In particular, the disturbance observer-based continuous finitetime sliding mode controller (DOBCSMC) is proposed to achieve the control objective under matched and mismatched disturbances, as stated in Section 2 (see Remark 1,).
4.1. Disturbance Observer-Based Continuous Finite-Time Sliding Mode Control

Assumption 1. The disturbances in (1) satisfy the following conditions: $d_{i}$ is differentiable twice and $\left|d_{i}^{(j)}\right| \leq L_{i}$ for $j=$ $0,1,2$ and $i=1,2$, where $L_{i}>0$.

Assumption 1 is inspired from $[25,26]$. This assumption is relatively common in various disturbance rejection control problems (see $[25,26]$ and the references therein). Specifically, various classes of disturbances such as highorder polynomial, sinusoidal, and cosinusoidal disturbances satisfy Assumption 1.

4.1.1. Finite-Time Disturbance Observer Design. Motivated by the work of Levant and Livne in [22], the nonlinear finite-time disturbance observer (NFTDO) is proposed as follows:

$$
\begin{aligned}
& \dot{z}_{0 j}=v_{0 j}+x_{j+1}, \\
& \dot{z}_{1 j}=v_{1 j}, \\
& \dot{z}_{2 j}=v_{2 j}, \\
& v_{0 j}=-\lambda_{0} L_{j}^{1 / 3}\left|z_{0 j}-x_{j}\right|^{2 / 3} \operatorname{sign}\left(z_{0 j}-x_{j}\right)-\varrho_{0}\left(z_{0 j}-x_{j}\right)+z_{1 j}, \\
& v_{1 j}=-\lambda_{1} L_{j}^{1 / 2}\left|z_{1 j}-v_{0 j}\right|^{1 / 2} \operatorname{sign}\left(z_{1 j}-v_{0 j}\right)-\varrho_{1}\left(z_{1 j}-v_{0 j}\right)+z_{2 j}, \\
& v_{2 j}=-\lambda_{2} L_{j} \operatorname{sign}\left(z_{2 j}-v_{1 j}\right)-\varrho_{2}\left(z_{2 j}-v_{1 j}\right),
\end{aligned}
$$

for $j=1,2$, and $x_{3}=f\left(x_{1}, x_{2}\right)+u$, where $\lambda_{0}, \lambda_{1}, \lambda_{2}$ and $\varrho_{0}, \varrho_{1}, \varrho_{2}$ are the observer coefficients to be designed, $z_{0 j}$ is the estimation of $x_{j}$, and $z_{1 j}, z_{2 j}$ are the estimation of $d_{j}, \dot{d}_{j}$.

Theorem 1. For system (1), if the NFTDO is designed as in (22) with the proper observer gains, then after a finite time, one can obtain $z_{0 j}=x_{j}, z_{1 j}=d_{j}, z_{2 j}=\dot{d}_{j}$, for $j=1,2$.

Proof. First, the estimation error variables are defined as follows:

$$
\begin{gathered}
\sigma_{0 j}=\frac{z_{0 j}-x_{j}}{L_{j}}, \\
\sigma_{1 j}=\frac{z_{1 j}-d_{j}}{L_{j}}, \\
\sigma_{2 j}=\frac{z_{2 j}-\dot{d}_{j}}{L_{j}} .
\end{gathered}
$$

It follows from (22) and (23) that 


$$
\begin{aligned}
\dot{\sigma}_{0 j}= & \frac{\dot{z}_{0 j}-\dot{x}_{j}}{L_{j}} \\
= & \frac{1}{L_{j}}\left(-\lambda_{0} L_{j}^{1 / 3}\left|L_{j} \sigma_{0 j}\right|^{2 / 3} \operatorname{sign}\left(\sigma_{0 j}\right)\right. \\
& \left.-\varrho_{0}\left(L_{j} \sigma_{0 j}\right)+z_{1 j}+x_{j+1}-x_{j+1}-d_{j}\right) \\
= & -\lambda_{0}\left|\sigma_{0 j}\right|^{2 / 3} \operatorname{sign}\left(\sigma_{0 j}\right)-\varrho_{0} \sigma_{0 j}+\sigma_{1 j}, \\
\dot{\sigma}_{1 j}= & \frac{z_{1 j}-\dot{d}_{j}}{L_{j}} \\
= & \frac{1}{L_{j}}\left(-\lambda_{1} L_{j}^{1 / 2}\left|z_{1 j}-v_{0 j}\right|^{1 / 2} \operatorname{sign}\left(z_{1 j}-v_{0 j}\right)\right. \\
& \left.-\varrho_{1}\left(z_{1 j}-v_{0 j}\right)+z_{2 j}-\dot{d}_{j}\right) \\
= & \frac{1}{L_{j}}\left(-\lambda_{1} L_{j}^{1 / 2}\left|z_{1 j}-v_{0 j}\right|^{1 / 2} \operatorname{sign}\left(z_{1 j}-v_{0 j}\right)\right. \\
& \left.-\varrho_{1}\left(z_{1 j}-v_{0 j}\right)\right)+\sigma_{2 j} \cdot
\end{aligned}
$$

It should be noted that

$$
\begin{aligned}
z_{1 j}-v_{0 j} & =\lambda_{0} L_{j}^{1 / 3}\left|z_{0 j}-x_{j}\right|^{2 / 3} \operatorname{sign}\left(z_{0 j}-x_{j}\right)+\varrho_{0}\left(z_{0 j}-x_{j}\right) \\
& =\lambda_{0} L_{j}^{1 / 3}\left|L_{j} \sigma_{0 j}\right|^{2 / 3} \operatorname{sign}\left(\sigma_{0 j}\right)+\varrho_{0}\left(L_{j} \sigma_{0 j}\right) \\
& =\lambda_{0} L_{j}\left|\sigma_{0 j}\right|^{2 / 3} \operatorname{sign}\left(\sigma_{0 j}\right)+\varrho_{0}\left(L_{j} \sigma_{0 j}\right) \\
& =L_{j}\left(\sigma_{1 j}-\dot{\sigma}_{0 j}\right) .
\end{aligned}
$$

Then, by substituting (26) into (25), we can obtain

$$
\begin{aligned}
\dot{\sigma}_{1 j}= & \frac{1}{L_{j}}\left\{-\lambda_{1} L_{j}^{1 / 2}\left|L_{j}\left(\sigma_{1 j}-\dot{\sigma}_{0 j}\right)\right|^{1 / 2} \operatorname{sign}\left(\sigma_{1 j}-\dot{\sigma}_{0 j}\right)\right. \\
& \left.-\varrho_{1}\left[L_{j}\left(\sigma_{1 j}-\dot{\sigma}_{0 j}\right)\right]\right\}+\sigma_{2 j} \\
= & -\lambda_{1}\left|\sigma_{1 j}-\dot{\sigma}_{0 j}\right|^{1 / 2} \operatorname{sign}\left(\sigma_{1 j}-\dot{\sigma}_{0 j}\right)-\varrho_{1}\left(\sigma_{1 j}-\dot{\sigma}_{0 j}\right)+\sigma_{2 j} .
\end{aligned}
$$

Following the same procedure as shown in (25)-(27), we can imply that

$$
\dot{\sigma}_{2 j}=-\lambda_{2} \operatorname{sign}\left(\sigma_{2 j}-\dot{\sigma}_{1 j}\right)-\varrho_{2}\left(\sigma_{2 j}-\dot{\sigma}_{1 j}\right)-\frac{1}{L_{j}} \ddot{d}_{j} .
$$

To summarize, the NFTDO estimation errors can be formed as

$$
\begin{aligned}
& \dot{\sigma}_{0 j}=-\lambda_{0}\left|\sigma_{0 j}\right|^{2 / 3} \operatorname{sign}\left(\sigma_{0 j}\right)-\varrho_{0} \sigma_{0 j}+\sigma_{1 j}, \\
& \dot{\sigma}_{1 j}=-\lambda_{1}\left|\sigma_{1 j}-\dot{\sigma}_{0 j}\right|^{1 / 2} \operatorname{sign}\left(\sigma_{1 j}-\dot{\sigma}_{0 j}\right)-\varrho_{1}\left(\sigma_{1 j}-\dot{\sigma}_{0 j}\right)+\sigma_{2 j}, \\
& \dot{\sigma}_{2 j}=-\lambda_{2} \operatorname{sign}\left(\sigma_{2 j}-\dot{\sigma}_{1 j}\right)-\varrho_{2}\left(\sigma_{2 j}-\dot{\sigma}_{1 j}\right)-\frac{1}{L_{j}} \ddot{d}_{j} .
\end{aligned}
$$

It follows from Lemma 1 that system (29) is finite-time stable, which implies that the estimation value converges to the actual value in finite time. This completes the proof of the theorem.

Remark 2. The readers are referred to the work of Levant and Livne [22] for a detailed guideline on the selection of observer gains $\lambda_{0}, \lambda_{1}, \lambda_{2}$ and $\varrho_{0}, \varrho_{1}, \varrho_{2}$. The trade-offs in the selection of observer parameters are as follows:

(i) The larger the values of $L_{j}$ and $\varrho_{j}$, the faster the convergence rate of the observer and the larger the peaking effect

(ii) The larger the value of $\lambda_{j}$, the faster the convergence rate of the observer and the higher the sensitivity to input noises and the sampling interval.

Remark 3. In comparison with the disturbance observer proposed in [7, 8], the proposed observer (22) provides faster convergence rate for large initial errors. This feature will be illustrated through the simulation results in Section 5 .

4.1.2. Continuous Finite-Time Sliding Mode Control. It should be noted that the system in (1) is subjected to both matched and mismatched disturbances. Based on the proposed observer, we develop the DOBCSMC to cope with matched and mismatched disturbances in system (1).

First, to reject the mismatched disturbance, a nonlinear sliding variable is defined as follows:

$$
s=\left(x_{2}+z_{11}\right)+\int_{0}^{t} k_{1}\left|x_{1}\right|^{\alpha_{1}} \operatorname{sign}\left(x_{1}\right)+k_{2}\left|x_{2}+z_{11}\right|^{\alpha_{2}} \operatorname{sign}\left(x_{2}+z_{11}\right) \mathrm{d} \tau,
$$

where the scalars $k_{1}$ and $k_{2}$ are selected such that the following polynomial $p^{2}+k_{2} p+k_{1}$ is Hurwitz and the scalars $\alpha_{1}$ and $\alpha_{2}$ are chosen as $\alpha_{1}=\alpha_{2} \alpha_{3} /\left(2 \alpha_{3}-\alpha_{2}\right)$ with $\alpha_{3}=1$ and $\alpha_{2}=\bar{\alpha} \in(0,1)$ and $z_{11}$ is the estimation of the mismatched disturbance $d_{1}$.

Then, the DOBCSMC is proposed as follows:

$$
\begin{aligned}
u= & -f\left(x_{1}, x_{2}\right)-v_{11}-z_{12}-\left[k_{1}\left|x_{1}\right|^{\alpha_{1}} \operatorname{sign}\left(x_{1}\right)\right. \\
& \left.+k_{2}\left|x_{2}+z_{11}\right|^{\alpha_{2}} \operatorname{sign}\left(x_{2}+z_{11}\right)\right] \\
& -\left[k_{3}|s|^{1 / 2} \operatorname{sign}(s)+\int_{0}^{t} k_{4} \operatorname{sign}(s) \mathrm{d} \tau\right],
\end{aligned}
$$

where $k_{3}$ and $k_{4}>0$ are the design parameters.

\section{Remark 4}

(i) The gains $\alpha_{1}$ and $\alpha_{2}$ need to be computed by simulations. The readers are referred to [27] for a detailed guideline on the selection of the sliding variable parameters $k_{1}, k_{2}, \alpha_{1}$, and $\alpha_{2}$ to ensure the finite-time convergence of the controlled output.

(ii) The values of $k_{3}$ and $k_{4}$ are selected to adjust the finite-time convergence to the sliding surface, in which with the large values of $k_{3}$ and $k_{4}$, the faster convergence rate can be obtained. However, they 
cannot be extremely large owing to the undesirable transient response.

4.2. Stability Analysis. We state the finite-time reachability and stability of the closed-loop system with the proposed DOBCSMC in (31).

Theorem 2. For the system in (1) with the proposed $D O B C S M C$ in (31), the controlled output $y$ converges to zero in finite time.

Proof. First, we obtain the closed-loop dynamics of the sliding variable (30) and system (1).

Taking the time derivative of the sliding variable (30) along the dynamics (1), we can obtain

$$
\begin{aligned}
\dot{s}= & \left(f\left(x_{1}, x_{2}\right)+u+d_{2}(t)+\dot{z}_{11}\right) \\
& +\left[k_{1}\left|x_{1}\right|^{\alpha_{1}} \operatorname{sign}\left(x_{1}\right)+k_{2}\left|x_{2}+z_{11}\right|^{\alpha_{2}} \operatorname{sign}\left(x_{2}+z_{11}\right)\right] .
\end{aligned}
$$
have

By substituting the control law given in (31) into (32), we

$$
\dot{s}=-k_{3}|s|^{1 / 2} \operatorname{sign}(s)-\int_{0}^{t} k_{4} \operatorname{sign}(s) \mathrm{d} \tau-e_{12},
$$

where $e_{12}$ is the auxiliary estimation error variable, which is defined as $e_{12}=z_{12}-d_{2}$.

The new state variables are defined as $\bar{x}_{1}=x_{1}, \bar{x}_{2}=x_{2}+z_{11}$. Then, the dynamics of the new states can be written as

$$
\begin{aligned}
& \dot{\bar{x}}_{1}=\bar{x}_{2}-e_{11}, \\
& \dot{\bar{x}}_{2}=-\left[k_{1}\left|\bar{x}_{1}\right|^{\alpha_{1}} \operatorname{sign}\left(\bar{x}_{1}\right)+k_{2}\left|\bar{x}_{2}\right|^{\alpha_{2}} \operatorname{sign}\left(\bar{x}_{2}\right)\right]+\dot{s},
\end{aligned}
$$

where $e_{11}$ is the auxiliary estimation error variable, which is defined as $e_{11}=z_{11}-d_{1}$.

We should note that both the sliding mode dynamics in (33) and the system dynamics in (34) are subjected to the error dynamics of the proposed observer given in (22).

Second, we show that the disturbance estimation error $e_{12}$ cannot drive the sliding variable $s$ to infinity in finitetime $T_{2}$, which denotes the convergence time of the proposed observer to estimate the matched disturbance $d_{2}$.

We define a function for (33) as follows:

$$
V_{1}(s)=\frac{1}{2} s^{2} .
$$

Below, we show that (35) is bounded in finite-time $T_{2}$. Taking the derivative of (35) along the dynamics (33), we have

$$
\begin{aligned}
\dot{V}_{1} & =s\left(-k_{3}|s|^{1 / 2} \operatorname{sign}(s)-\int_{0}^{t} k_{4} \operatorname{sign}(s) \mathrm{d} \tau-e_{12}\right) \\
& =-k_{3}|s|^{3 / 2}-k_{4} s \int_{0}^{t} \operatorname{sign}(s) \mathrm{d} \tau-s e_{12} \\
& \leq k_{4}|s| \int_{0}^{t}|\operatorname{sign}(s)| \mathrm{d} \tau+|s|\left|e_{12}\right| .
\end{aligned}
$$

By using Lemma 4 , we can obtain for $t \in\left[0, T_{2}\right]$

$$
\begin{aligned}
\dot{V}_{1} & \leq k_{4} T_{2}\left(\frac{1}{2} s^{2}+\frac{1}{2}\right)+\left|e_{12}\right|\left(\frac{1}{2} s^{2}+\frac{1}{2}\right) \\
& \leq K_{\max } V_{1}+L_{\max },
\end{aligned}
$$

where $\quad K_{\max }=\max \left\{k_{4} T_{2}+\left|e_{12}\right|\right\} \quad$ and $\quad L_{\max }=(1 / 2)$ $\max \left\{k_{4} T_{2}+\left|e_{12}\right|\right\}$ are bounded constants owing to the boundness of $e_{12}$ in view of Theorem 1 .

Therefore, it can be concluded from (37) that $V_{1}$ and so $s$ will not escape to infinity in finite-time $T_{2}$. Furthermore, the estimation error $e_{12}$ will converge to zero in a finite-time $T_{2}$. Hence, combining the fact that sliding variable $s$ will not go to infinity in finite-time $T_{2}$, the sliding mode dynamics (33) can be reduced to

$$
\dot{s}=-k_{3}|s|^{1 / 2} \operatorname{sign}(s)-\int_{0}^{t} k_{4} \operatorname{sign}(s) \mathrm{d} \tau,
$$

when $t \geq T_{2}$.

It should be noted that (38) is in the form of the supertwisting algorithm. Hence, in light of Lemma 2, we can conclude that $s$ and $\dot{s}$ will converge to zero in finitetime $T_{s}$.

Third, we show that the estimation error $e_{11}$ and the derivative of sliding variable $\dot{s}$ will not drive the system states $\bar{x}_{1}$ and $\bar{x}_{2}$ to infinity in finite-time $T=\max \left\{T_{1}, T_{s}\right\}$, where $T_{1}$ denotes the convergence time of the proposed observer to estimate the mismatched disturbance $d_{1}$.

We define a function for (34) as follows:

$$
V_{2}\left(\bar{x}_{1}, \bar{x}_{2}\right)=\frac{1}{2} \bar{x}_{1}^{2}+\frac{1}{2} \bar{x}_{2}^{2} \text {. }
$$

Below, we show that (39) is bounded in finite-time $T$. Taking the derivative of (39) along the dynamics (34), we have

$$
\begin{aligned}
\dot{V}_{2} & =\bar{x}_{1}\left(\bar{x}_{2}-e_{11}\right)+\bar{x}_{2}\left\{-\left[k_{1}\left|\bar{x}_{1}\right|^{\alpha_{1}} \operatorname{sign}\left(\bar{x}_{1}\right)+k_{2}\left|\bar{x}_{2}\right|^{\alpha_{2}} \operatorname{sign}\left(\bar{x}_{2}\right)\right]+\dot{s}\right\} \\
& =\bar{x}_{1} \bar{x}_{2}-\bar{x}_{1} e_{11}-k_{1} \bar{x}_{2}\left|\bar{x}_{1}\right|^{\alpha_{1}} \operatorname{sign}\left(\bar{x}_{1}\right)-k_{2} \bar{x}_{2}\left|\bar{x}_{2}\right|^{\alpha_{2}} \operatorname{sign}\left(\bar{x}_{2}\right)+\bar{x}_{2} \dot{s} .
\end{aligned}
$$

By using Lemma 4 and the inequality $\left|\bar{x}_{1}\right|^{\alpha_{1}}<1+\left|\bar{x}_{1}\right|$ for $0<\alpha_{1}<1$, we can obtain for $t \in[0, T]$,

$$
\begin{aligned}
\dot{V}_{2} \leq & \left|\bar{x}_{1}\right|\left|\bar{x}_{2}\right|+\left|\bar{x}_{1}\right|\left|e_{11}\right|+k_{1}\left|\bar{x}_{2}\right|\left(1+\left|\bar{x}_{1}\right|\right)+k_{2}\left|\bar{x}_{2}\right|\left(1+\left|\bar{x}_{2}\right|\right)+\left|\bar{x}_{2}\right||\dot{s}| \\
\leq & \left(\frac{1}{2} \bar{x}_{1}^{2}+\frac{1}{2} \bar{x}_{2}^{2}\right)+\left(\frac{1}{2} \bar{x}_{1}^{2}+\frac{1}{2} e_{11}^{2}\right)+\left(\frac{1}{2} \bar{x}_{2}^{2}+\frac{1}{2}\left(k_{1}+k_{2}\right)^{2}\right)+k_{1}\left(\frac{1}{2} \bar{x}_{1}^{2}+\frac{1}{2} \bar{x}_{2}^{2}\right) \\
& +k_{2}\left(\frac{1}{2} \bar{x}_{2}^{2}+\frac{1}{2} \bar{x}_{2}^{2}\right)+\left(\frac{1}{2} \bar{x}_{2}^{2}+\frac{1}{2} \dot{s}^{2}\right) \\
\leq & K_{2 \max } V_{2}+L_{2 \max },
\end{aligned}
$$

where $K_{2 \max }=3+k_{1}+2 k_{2}$ and $L_{2 \max }=(1 / 2) \max \left\{\left(k_{1}+\right.\right.$ $\left.\left.k_{2}\right)^{2}+e_{11}^{2}+\dot{s}^{2}\right\}$ are bounded constants owing to the boundness of $e_{11}$ in view of Theorem 1 and the boundness of $\dot{s}$ in view of (33). Therefore, it can be concluded from (41) that $V_{2}$ is bounded, which implies that the state variables $\bar{x}_{1}$ and $\bar{x}_{2}$ will not escape to infinity in finite-time $T$. 
We now show that the controlled output $y=x_{1}$ converges to zero in finite time. Once the finite-time convergence of the proposed observer and the sliding mode dynamics are achieved, system dynamics (34) is reduced as follows:

$$
\begin{aligned}
& \dot{\bar{x}}_{1}=\bar{x}_{2}, \\
& \dot{\bar{x}}_{2}=-\left[k_{1}\left|\bar{x}_{1}\right|^{\alpha_{1}} \operatorname{sign}\left(\bar{x}_{1}\right)+k_{2}\left|\bar{x}_{2}\right|^{\alpha_{2}} \operatorname{sign}\left(\bar{x}_{2}\right)\right] .
\end{aligned}
$$

Then, it follows from Lemma 3 that the system in (42) is finite-time stable, which implies that the controlled output $x_{1}$ converges to zero in finite time. This completes the proof of the theorem.

\section{Remark 5}

(i) We note that it is not possible to use directly the traditional SMC method [1-3] or even the secondorder finite-time SMC approach mentioned in Lemmas 2 and 3 to guarantee the stability of the controlled output of the system in (1). Such mismatched disturbances need to be estimated first, and then the SMC control technique can be applied based on the disturbance estimation to compensate for the effect of the disturbance on the controlled output.

(ii) Besides, our stability proof is different from that of $[4,5]$. Specially, in our proof, we first need to prove that the sliding variable and the system states will not diverge to infinity during the convergence period of the proposed disturbance observer. Then, the finitetime stability of the controlled output is shown by means of the proposed sliding variable and corresponding continuous SMC control law.

Remark 6 (nominal performance recovery). In the absence of disturbance, it is derived from Theorem 1 that $z_{0 j}(t)=$ $x_{j}(t)$ and $z_{1 j}(t)=z_{2 j}(t)=0$ if the initial values of the observer states are selected as $z_{0 j}\left(t_{0}\right)=x_{j}\left(t_{0}\right)$ and $z_{1 j}\left(t_{0}\right)=z_{2 j}\left(t_{0}\right)=0$. In this case, the proposed DOBCSMC is reduced to the supertwisting algorithm. This implies that the nominal control performance of the proposed method is retained. Such interesting features will be demonstrated in the numerical example in Section 5.

Remark 7. The results obtained in this paper can be extended to the $n$ th-order input-affine nonlinear system described by

$$
\begin{aligned}
\dot{x}_{i} & =x_{i+1}+d_{i}(t), \quad i \\
\dot{x}_{n} & =f(x)+u+d_{n}(t), \\
y & =x_{1},
\end{aligned}
$$

where $x=\left[x_{1}, \ldots, x_{n}\right]^{T} \in \mathbb{R}^{n}$ is the system state vector, $u$ is the control input, $y$ is the controlled output, $f$ is a smooth nonlinear function, and $d_{i}$ and $d_{n}$ denote the mismatched and matched disturbances, respectively. In this case, we first need to convert the $n$ th-order system into the equivalent system with different state variables similar to (34). Then, the estimations of the mismatched disturbance and its derivatives have to be included in the sliding surface and the control law appropriately.

\section{Simulation Results}

This section provides the simulation results to validate the theoretical results obtained in Section 4.

We consider the following system, which can be viewed as a modification of $[6,28,29]$ :

$$
\begin{aligned}
& \dot{x}_{1}=x_{2}+d_{1}(t), \\
& \dot{x}_{2}=-2 x_{1}-x_{2}+e^{x_{1}}+u+d_{2}(t), \\
& y=x_{1} .
\end{aligned}
$$

For verification, we consider the following three different simulation scenarios:

(i) Time-Invariant Disturbance Rejection Performance (Scenario I). We demonstrate the time-invariant disturbance rejection performance of the proposed DOBCSMC by comparing the results with those of the ESMC in [6], the CDSMC in [7], and the CNTSMC in [8].

(ii) Time-Varying Disturbance Rejection Performance (Scenario II). The control performance of the ESMC, CDSMC, CNTSMC, and the proposed DOBCSMC is considered under the effect of the time-varying matched and mismatched disturbances.

(iii) Nominal Performance Recovery (Scenario III). We consider the nominal control performance recovery of the proposed DOBCSMC in the absence of disturbances.

5.1. Time-Invariant Disturbance Rejection Performance (Scenario I). In this section, we verify the performance of the ESMC, CDSMC, CNTSMC, and the proposed DOBCSMC in the presence of the time-invariant mismatched disturbance.

We take $x(0)=[1,-1]^{T}$ in (44). The mismatched and matched disturbances are set as $d_{1}(t)=0.5$ and $d_{2}(t)=0$ [6]. To make a fair comparison (e.g., convergence rate, control accuracy, and chattering alleviation), the limited control input is selected as \pm 40 .

As illustrated in Figure 1, we can observe that the controlled output converges to zero in the presence of a time-invariant mismatched disturbance with all four control methods. Moreover, from Figures 1 and 2, we can see that the proposed DOBCSMC can ensure the highest control accuracy with less overshoot and the fastest convergence rate, compared with the ESMC, CDSMC, and CNTSMC. These properties are obtained owing to the utilization of the proposed nonlinear observer together with the nonlinear sliding variable and the supertwisting algorithm.

To observe the effectiveness of the proposed nonlinear finite-time observer compared with the observer used in the CDSMC and CNTSMC, we provide the disturbance estimation error, as shown in Figure 3. It is evident that both 


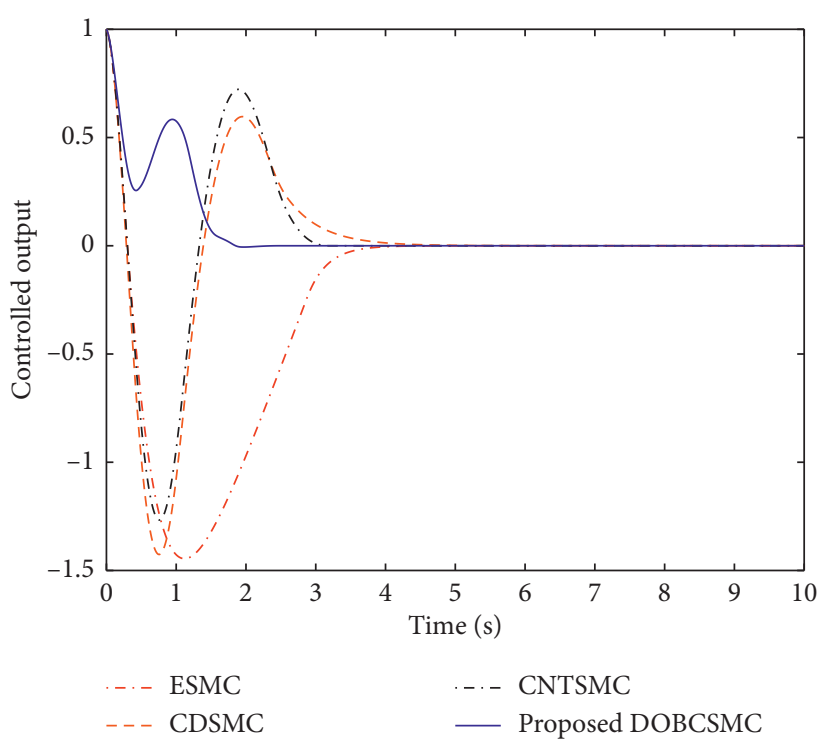

(a)

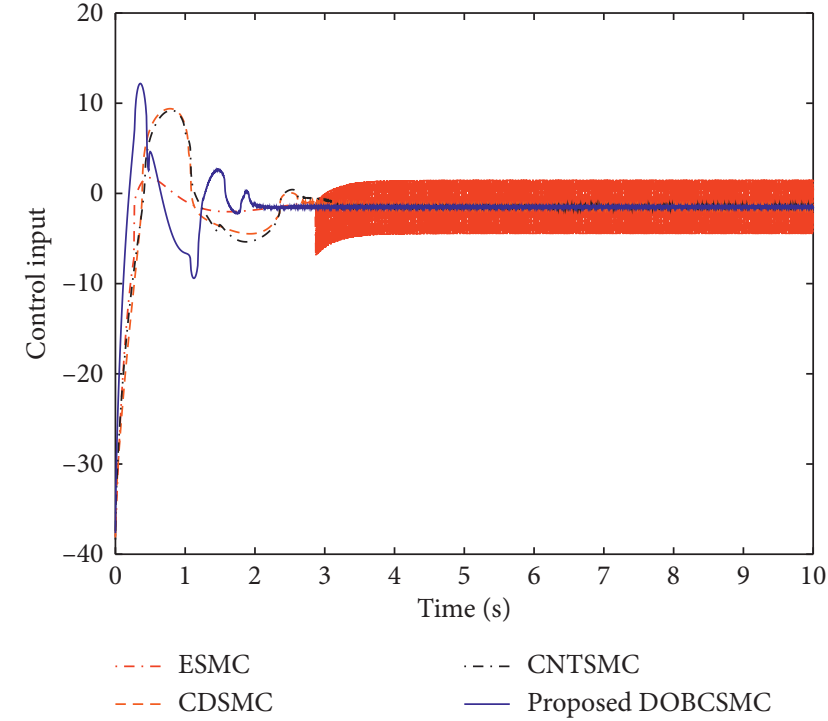

(b)

Figure 1: Simulation results of Scenario I.

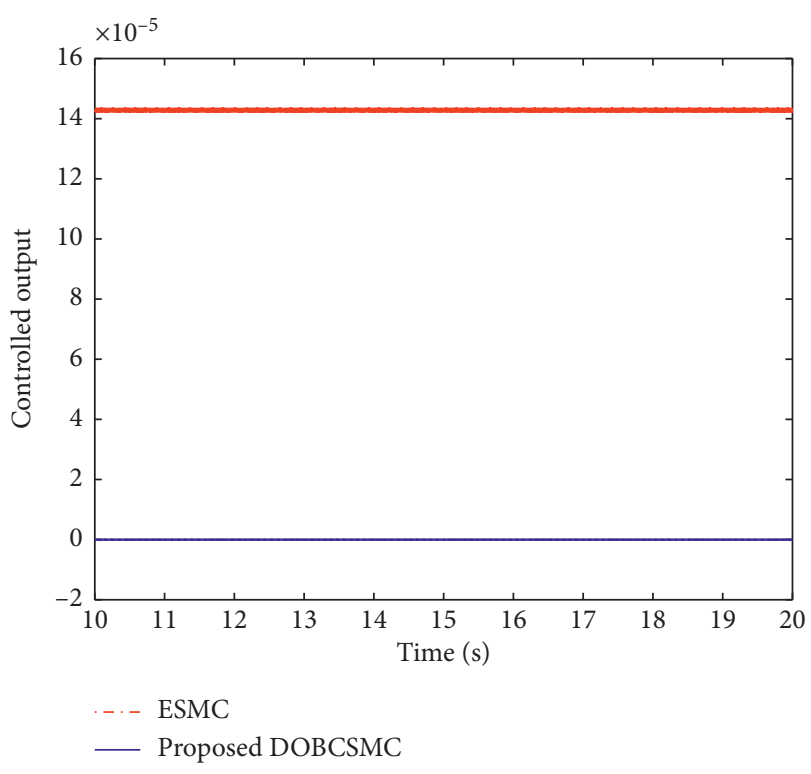

(a)

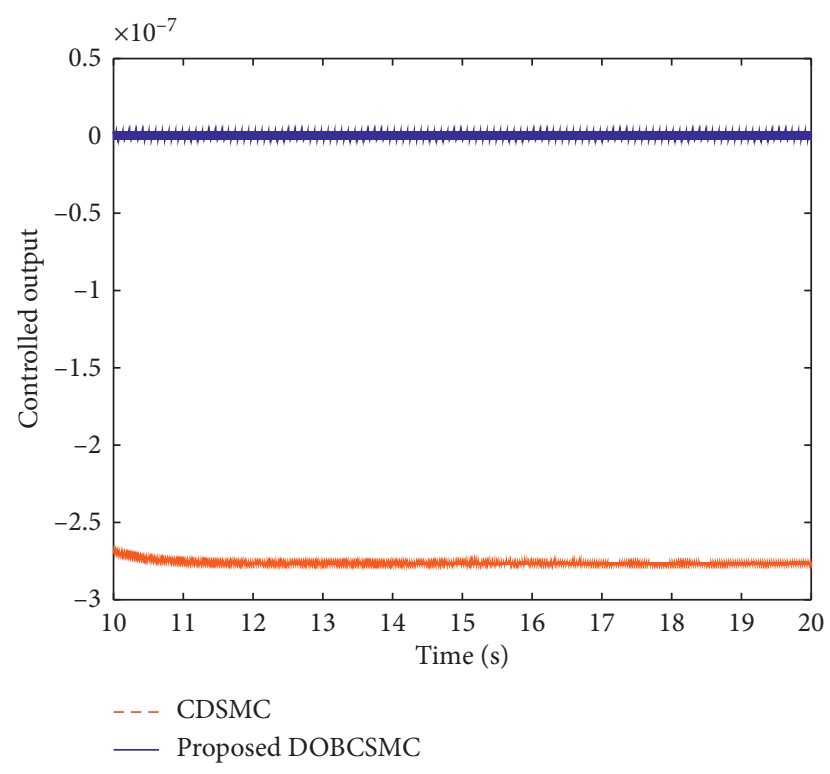

(b)

FIgURe 2: Continued. 


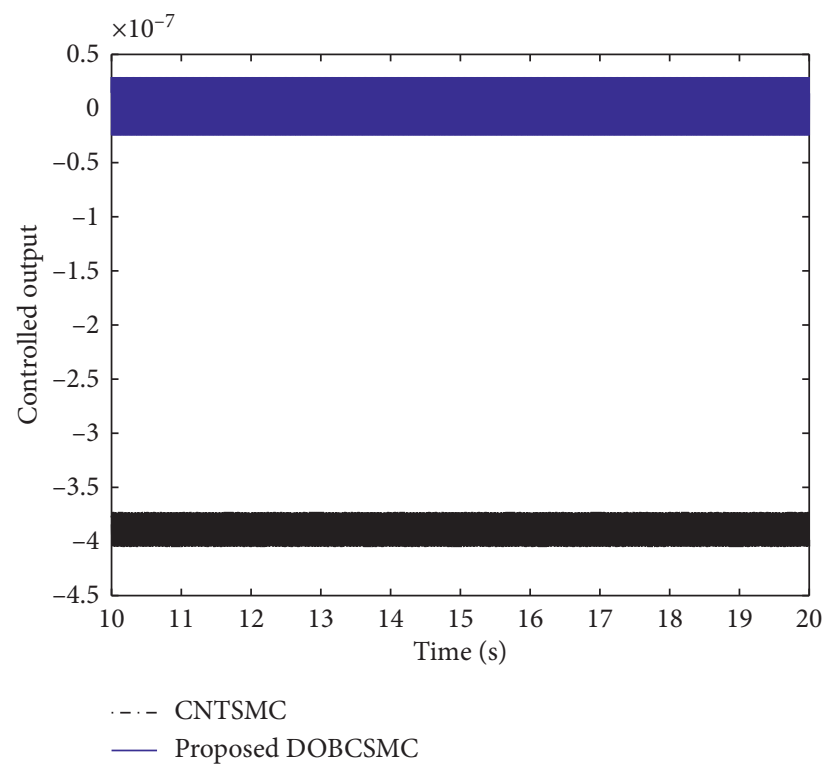

(c)

FIGURE 2: Simulation results of Scenario I-controlled output in steady-state phase.

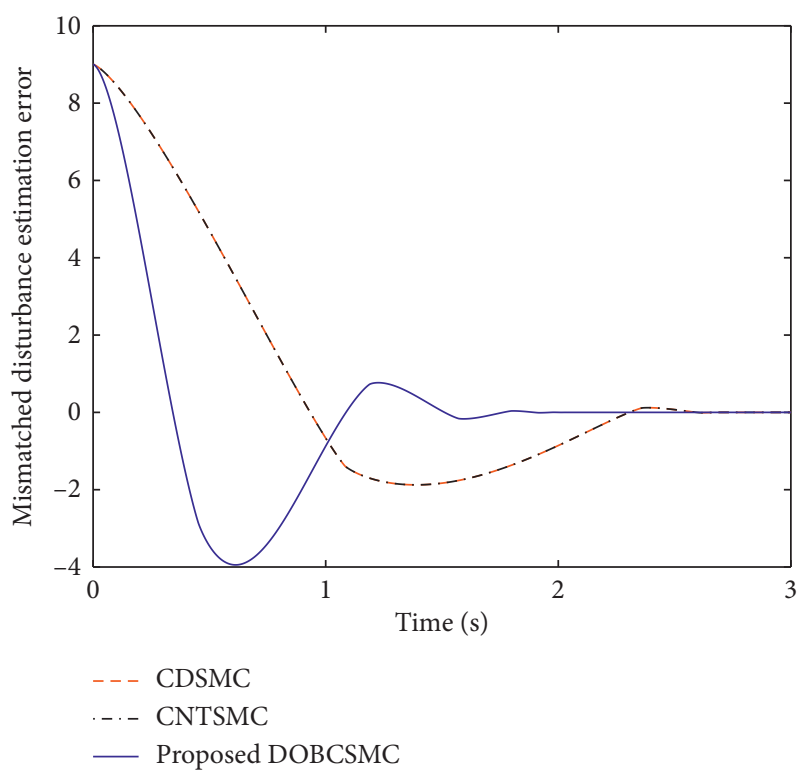

FIGURE 3: Simulation results of Scenario I-mismatched disturbance estimation error.

observers can track the disturbances accurately, but our proposed observer provides faster settling time. This result verifies the statement in Remark 3.

The detailed comparison of the control performance for Scenario I with different controllers is presented in Table 2. In Table 2, the system state error (SE) is the region when $x_{1}$ is in steady state. The convergence time (CT) is the time after which $\left|x_{1}\right| \leq 0.01$ always holds. Although ESMC, CDSMC, CNTSMC, and DOBCSMC show similar steady state error, the DOBCSMC provides the fastest convergence time.

From the simulation results of this section, we can conclude that the proposed DOBCSMC provides better control
TABLE 2: Control performance of ESMC, CDSMC, CNTSMC, and DOBCSMC for Scenario I.

\begin{tabular}{lcccc}
\hline & ESMC & CDSMC & CNTSMC & DOBCSMC \\
\hline SE & $\pm 1.4 \times 10^{-4}$ & $\pm 2.8 \times 10^{-6}$ & $\pm 4.0 \times 10^{-7}$ & $\pm 3.0 \times 10^{-8}$ \\
CT (s) & 3.779 & 4.144 & 3.004 & 1.795 \\
\hline
\end{tabular}

performance and faster convergence rate of the controlled output compared with ESMC, CDSMC, and CNTSMC, which confirms the statement in Introduction and Remark 1.

5.2. Time-Varying Disturbance Rejection Performance (Scenario II). In this section, we verify the performance of ESMC, CDSMC, CNTSMC, and the proposed DOBCSMC in the presence of time-varying matched and mismatched disturbances. We choose the mismatched and matched disturbances as $d_{1}(t)=2+\sin (t)-0.5 \cos (t)$ and $d_{2}(t)=\sin (t)+\sin (2 t)$. The other parameters are the same as those in Section 5.1 .

As illustrated in Figure 4, the controlled output $y=x_{1}$ cannot converge to zero with the ESMC. This is because the ESMC cannot handle the time-varying mismatched disturbance, as stated in Introduction and Section 2.3. It should be noted that CDSMC, CNTSMC, and the proposed DOBCSMC can reject (or attenuate) both time-invariant and time-varying mismatched disturbances as shown in Figure 4. However, the proposed DOBCSMC provides better control accuracy and the fastest convergence rate, compared with CDSMC and CNTSMC. A detailed comparison of the control performance for Scenario II with different controllers is presented in Table 3. Although CDSMC, CNTSMC, and DOBCSMC show similar steady state error, the proposed DOBCSMC provides the fastest convergence time. 


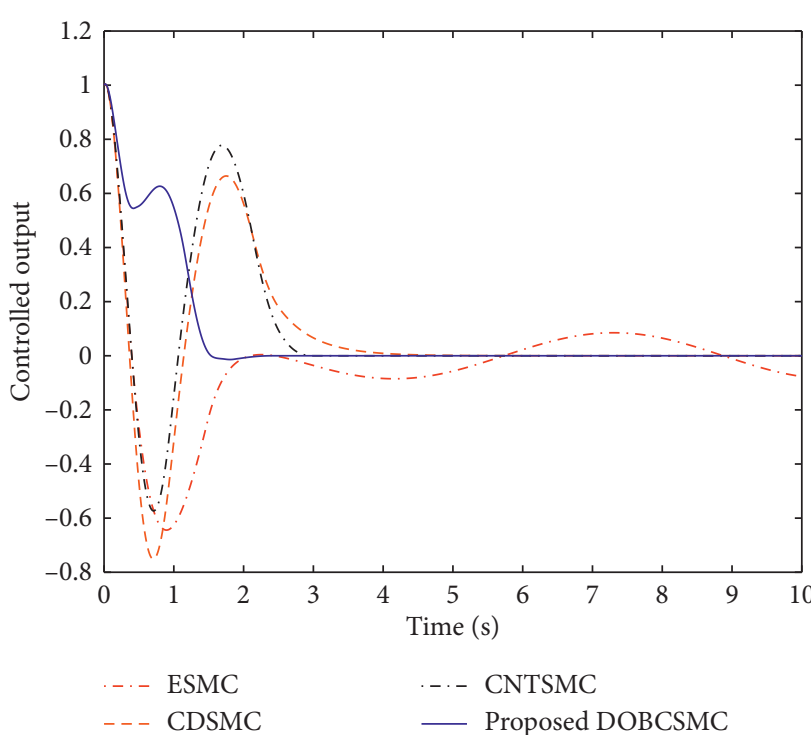

(a)

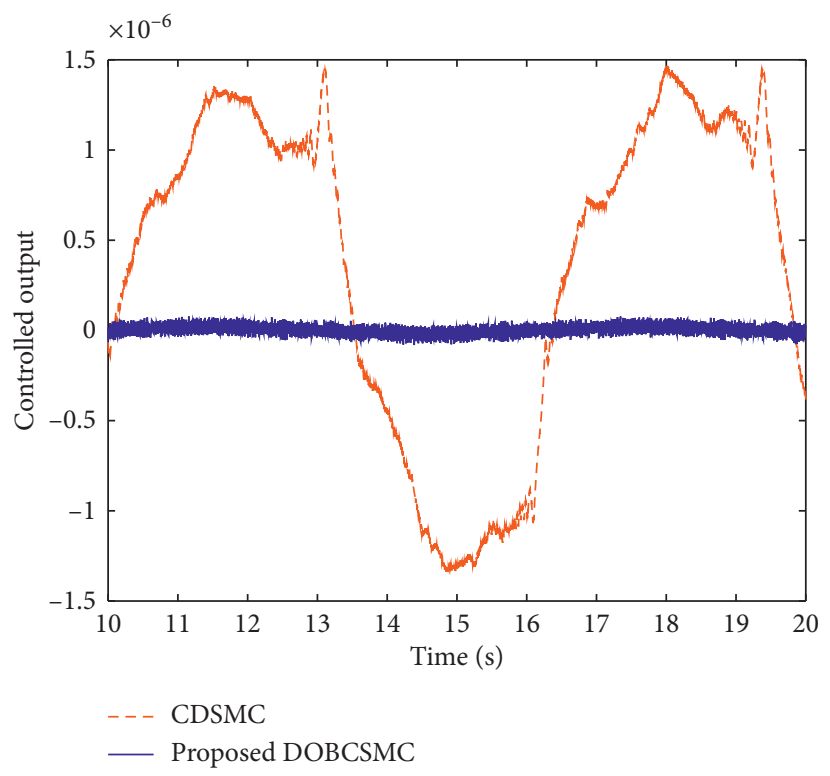

(c)

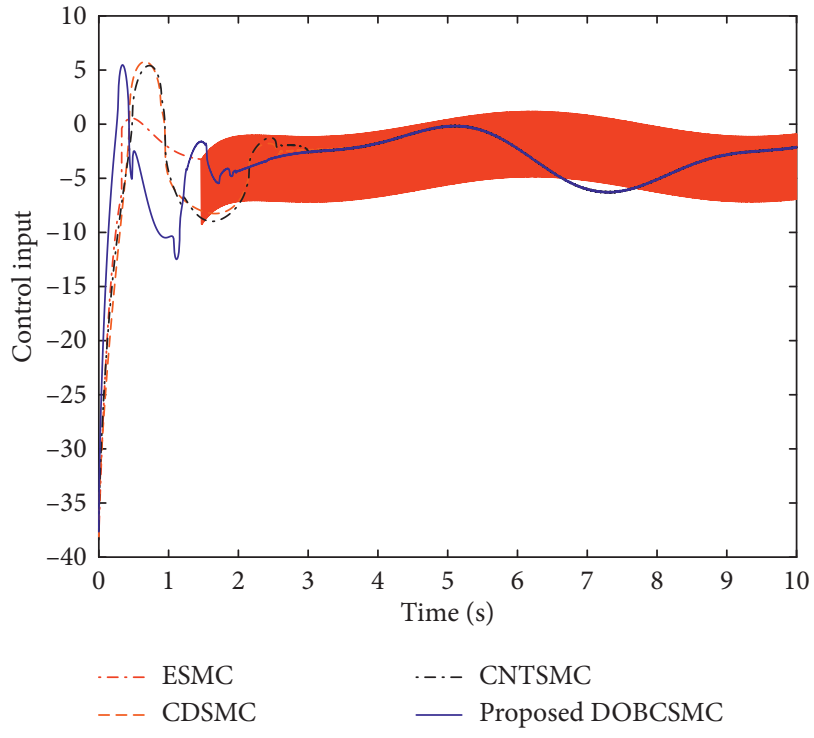

(b)

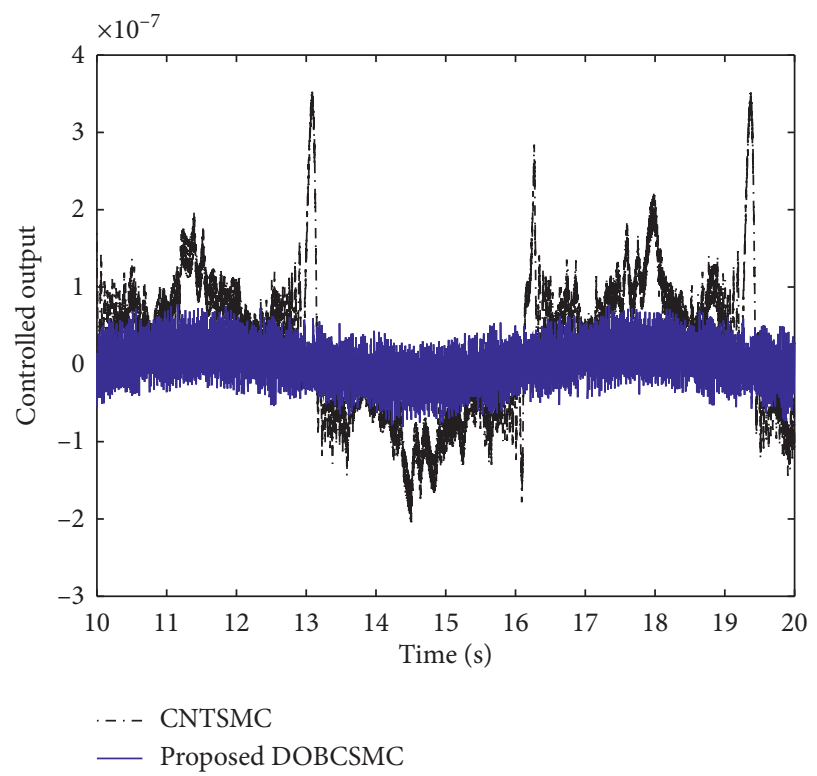

(d)

Figure 4: Continued. 


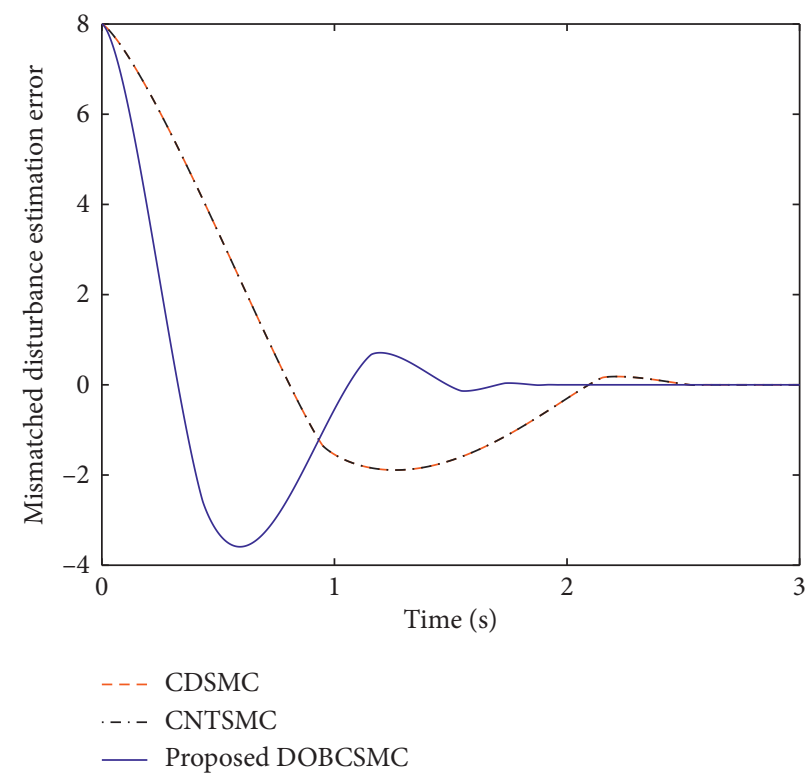

(e)

FIgURE 4: Simulation results of Scenario II.

TABLE 3: Control performance of ESMC, CDSMC, CNTSMC, and DOBCSMC for Scenario II.

\begin{tabular}{lcccc}
\hline & ESMC & CDSMC & CNTSMC & DOBCSMC \\
\hline SE & N/A & $\pm 1.5 \times 10^{-6}$ & $\pm 3.5 \times 10^{-7}$ & $\pm 8.0 \times 10^{-8}$ \\
CT $(\mathrm{s})$ & N/A & 3.947 & 2.798 & 1.499 \\
\hline
\end{tabular}

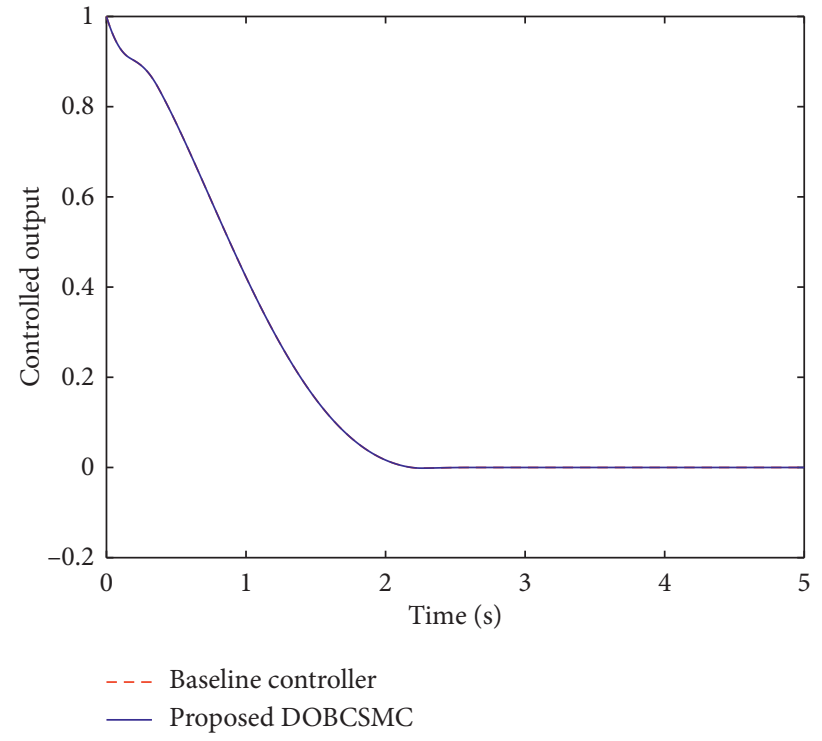

(a)

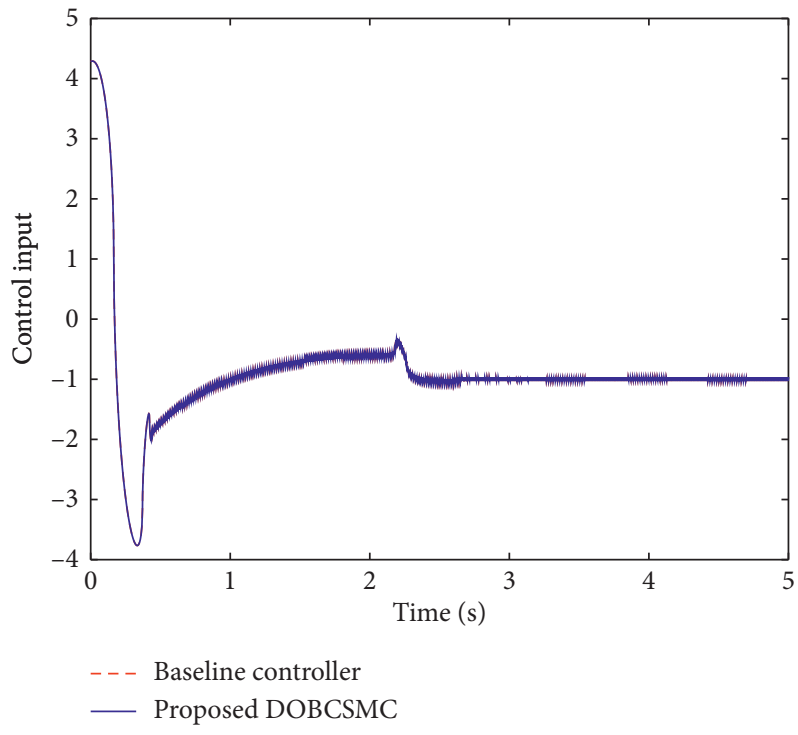

(b)

FIgure 5: Nominal performance recovery with the DOBCSMC (Scenario III).

Therefore, we can claim that the proposed DOBCSMC provides better disturbance attenuation and control performance, compared with ESMC, CDSMC, and CNTSMC, which confirms the statement in Introduction and Remark 1.
5.3. Nominal Performance Recovery (Scenario III). We evaluate the nominal performance recovery feature of the proposed DOBCSMC in the absence of disturbances, i.e., $d_{1}(t)=d_{2}(t)=0$. The response curves of the controlled 
output under the proposed DOBCSMC and the baseline controller are illustrated in Figure 5.

We can observe that the system states are mostly the same, which is the evidence that the nominal performance of the proposed DOBCSMC is retained. This result verifies the statement in Remark 6.

\section{Conclusions}

In this paper, we have proposed the disturbance observerbased continuous finite-time sliding mode controller to attenuate (or reject) both time-varying matched and mismatched disturbances for a class of input-affine nonlinear systems. We have shown that the closed-loop system with the proposed DOBCSMC guarantees both finite-time reachability to the sliding surface and finite-time stability of the controlled output to the origin. Various simulation results have been provided, which show the effectiveness of the proposed DOBCSMC in terms of disturbance attenuation and chattering reduction, compared with the existing $\mathrm{SMC}$ approaches. One possible future research direction is to extend the results of the paper to a class of underactuated systems with matched and mismatched disturbances. Another possible future research direction is to extend the proposed DOBCSMC to high-order systems subjected to matched and mismatched disturbances as discussed in Remark 7.

\section{Data Availability}

No data were used to support this study.

\section{Conflicts of Interest}

The authors declare that they have no conflicts of interest.

\section{Acknowledgments}

This research was supported by the National Research Foundation of Korea (NRF), funded by the Ministry of Science and ICT (NRF-2017R1A5A1015311).

\section{References}

[1] C. Edwards and S. Spurgeon, Sliding Mode Control: Theory and Applications, CRC Press, New York, NY, USA, 1998.

[2] W. Perruquetti and J.-P. Barbot, Sliding Mode Control in Engineering, CRC Press, New York, NY, USA, 2002.

[3] V. I. Utkin, Sliding Modes in Control and Optimization, Springer Science \& Business Media, New York, NY, USA, 2013.

[4] J. A. Moreno and M. Osorio, "A Lyapunov approach to second-order sliding mode controllers and observers," in Proceedings of the 47th IEEE Conference on Decision and Control, pp. 2856-2861, Cancun, Mexico, USA, December 2008.

[5] S. P. Bhat and D. S. Bernstein, "Geometric homogeneity with applications to finite-time stability," Mathematics of Control, Signals, and Systems, vol. 17, no. 2, pp. 101-127, 2005.

[6] J. Yang, S. Li, and X. Yu, "Sliding-mode control for systems with mismatched uncertainties via a disturbance observer,"
IEEE Transactions on Industrial Electronics, vol. 60, no. 1, pp. 160-169, 2012.

[7] J. Yang, J. Su, S. Li, and X. Yu, "High-order mismatched disturbance compensation for motion control systems via a continuous dynamic sliding-mode approach," IEEE Transactions on Industrial Informatics, vol. 10, no. 1, pp. 604-614, 2013.

[8] J. Yang, S. Li, J. Su, and X. Yu, "Continuous nonsingular terminal sliding mode control for systems with mismatched disturbances," Automatica, vol. 49, no. 7, pp. 2287-2291, 2013.

[9] V. Utkin and J. Shi, "Integral sliding mode in systems operating under uncertainty conditions," in Proceedings of the 35th IEEE Conference on Decision and Control, pp. 4591-4596, Kobe, Japan, December 1996.

[10] W.-J. Cao and J.-X. Xu, "Nonlinear integral-type sliding surface for both matched and unmatched uncertain systems," IEEE Transactions on Automatic Control, vol. 49, no. 8, pp. 1355-1360, 2004.

[11] M. Rubagotti, A. Estrada, F. Castanos, A. Ferrara, and L. Fridman, "Integral sliding mode control for nonlinear systems with matched and unmatched perturbations," IEEE Transactions on Automatic Control, vol. 56, no. 11, pp. 2699-2704, 2011.

[12] J. Lian, J. Zhao, and G. M. Dimirovski, "Integral sliding mode control for a class of uncertain switched nonlinear systems," European Journal of Control, vol. 16, no. 1, pp. 16-22, 2010.

[13] Y. Chang, "Adaptive sliding mode control of multi-input nonlinear systems with perturbations to achieve asymptotical stability," IEEE Transactions on Automatic Control, vol. 54, no. 12, pp. 2863-2869, 2009.

[14] J. Zhang, P. Shi, and Y. Xia, "Robust adaptive sliding-mode control for fuzzy systems with mismatched uncertainties," IEEE Transactions on Fuzzy Systems, vol. 18, no. 4, pp. 700711, 2010.

[15] A. Estrada and L. Fridman, "Quasi-continuous HOSM control for systems with unmatched perturbations," Automatica, vol. 46, no. 11, pp. 1916-1919, 2010.

[16] H. Y. Li and Y. A. Hu, "Robust sliding-mode backstepping design for synchronization control of cross-strict feedback hyperchaotic systems with unmatched uncertainties," Communications in Nonlinear Science and Numerical Simulation, vol. 16, no. 10, pp. 3904-3913, 2011.

[17] H. Zhang, Q. Qu, G. Xiao, and Y. Cui, "Optimal guaranteed cost sliding mode control for constrained-input nonlinear systems with matched and unmatched disturbances," IEEE Transactions on Neural Networks and Learning Systems, vol. 29, no. 6, pp. 2112-2126, 2018.

[18] M. Van, S. S. Ge, and H. Ren, "Finite time fault tolerant control for robot manipulators using time delay estimation and continuous nonsingular fast terminal sliding mode control," IEEE Transactions on Cybernetics, vol. 47, no. 7, pp. 1681-1693, 2016.

[19] K. Devika and S. Thomas, "Power rate exponential reaching law for enhanced performance of sliding mode control," International Journal of Control, Automation and Systems, vol. 15, no. 6, pp. 2636-2645, 2017.

[20] Y. Feng, F. Han, and X. Yu, "Chattering free full-order slidingmode control," Automatica, vol. 50, no. 4, pp. 1310-1314, 2014.

[21] Z. Song, C. Duan, H. Su, and J. Hu, "Full-order sliding mode control for finite-time attitude tracking of rigid spacecraft," IET Control Theory \& Applications, vol. 12, no. 8, pp. 10861094, 2018. 
[22] A. Levant and M. Livne, "Globally convergent differentiators with variable gains," International Journal of Control, vol. 91, no. 9, pp. 1994-2008, 2018.

[23] A. Levant, "Non-homogeneous finite-time-convergent differentiator," in Proceedings of the 48h IEEE Conference on Decision and Control (CDC) Held Jointly with the 28th Chinese Control Conference, pp. 8399-8404, Shanghai, China, December 2009.

[24] Y. Hua, X. Dong, L. Han, Q. Li, and Z. Ren, "Finite-time timevarying formation tracking for high-order multiagent systems with mismatched disturbances," IEEE Transactions on Systems, Man, and Cybernetics: Systems, pp. 1-9, 2019.

[25] J. Yang, Z. Ding, S. Li, and C. Zhang, "Continuous finite-time output regulation of nonlinear systems with unmatched timevarying disturbances," IEEE Control Systems Letters, vol. 2, no. 1, pp. 97-102, 2017.

[26] C. Zhang, J. Yang, Y. Yan, L. Fridman, and S. Li, "Semi-global finite-time trajectory tracking realization for disturbed nonlinear systems via higher-order sliding modes," IEEE Transactions on Automatic Control, vol. 65, no. 5, pp. 2185-2191, 2020.

[27] C. Edwards and Y. Shtessel, "Enhanced continuous higher order sliding mode control with adaptation," Journal of the Franklin Institute, vol. 356, no. 9, pp. 4773-4784, 2019.

[28] D. Ginoya, P. Shendge, and S. Phadke, "Sliding mode control for mismatched uncertain systems using an extended disturbance observer," IEEE Transactions on Industrial Electronics, vol. 61, no. 4, pp. 1983-1992, 2013.

[29] S.-L. Shi, J.-X. Li, and Y.-M. Fang, "Extended-state-observerbased chattering free sliding mode control for nonlinear systems with mismatched disturbance," IEEE Access, vol. 6, pp. 22952-22957, 2018. 